\title{
Probing the transport properties of each individual wall within a multiwall carbon nanotubes by electric breakdown
}

\author{
J. Y. Huang,* S. Chen,* Z. F. Ren,* Z. Q. Wang,* K. Kempa,* M. J. Naughton,* G. Chen,** \\ and M. S. Dresselhaus*** \\ *Department of Physics, Boston College, Chestnut Hill, MA 02467, USA \\ **Department of Mechanical Engineering, Massachusetts Institute of Technology, Cambridge, \\ Massachusetts 02139, USA \\ ***Department of Physics, Electrical Engineering and Computer Science, Massachusetts Institute of \\ Technology, Cambridge, Massachusetts 02139, USA
}

Conventional transport studies of multiwall carbon nanotubes (MWCNTs) with only the outmost wall contacted to the electrode via side-contact (Fig. 1a) shows that a MWCNT is a ballistic conductor with only the outmost wall carrying current. Here we report, using end-contact (Fig. 1b) and in high vacuum, the atomic-scale imaging with concurrent transport measurements of the wallby-wall breakdown of individual MWCNTs inside a high resolution transmission electron microscope (HRTEM) equipped with a scanning tunneling microscope (Nanofactory TEM-STM system). We found unexpectedly three distinct breakdown sequences, namely, from the outermost wall inward, from the innermost wall outward, and alternatively between the innermost and the outmost walls [1]. Remarkably, a significant amount of current drop was observed when an innermost wall is broken, proving unambiguously that every wall is conducting, and a MWCNT is the smallest parallel resistor. Moreover, the breakdown of each wall in any sequence initiates in the middle of the nanotube, not at the contact, proving that the transport is not ballistic but diffusive.

Figure 2 shows that a six-wall nanotube was peeled off layer-by-layer in a sequence from the outmost wall to the innermost wall by high-bias induced thermal breakdown [1]. When passing a current of $240 \mu \mathrm{A}$, breakdown occurs midway of the MWCNT, resulting in the formation of a clean 6-wall nanotube segment, which was eliminated wall-by-wall. The loss of one wall under a constant bias of $3 \mathrm{~V}$ results in an instant current drop (Fig. 2f). The current drops are approximately: 13, 17, 25 , and $31 \mu \mathrm{A}$ for the $6^{\text {th }}, 5^{\text {th }}, 4^{\text {th }}$, and $3^{\text {rd }}$ wall breakdown (the innermost wall is labeled as the $1^{\text {st }}$ wall), respectively. HRTEM indicates that the breakdown eventually results in the formation of either a double-wall nanotube or a single-wall nanotube junction [1-3]. Remarkably, HRTEM detects that each breakdown initiates in the middle of the nanotube, not at the contact (Fig. 2).

The temperature distribution in the nanotubes is parabolic, with the highest temperature being in the middle of the nanotube, which is estimated to be over $2000{ }^{\circ} \mathrm{C}$ at the breakdown voltage of $3 \mathrm{~V}$. We suggest that the breakdown is triggered by resistive heating, thus it is not difficult to understand the breakdown occurs in the middle of the nanotube, starting either from the outermost wall or from the innermost wall.

References

[1] J. Y. Huang et al., Phys. Rev. Lett. 94 (2005) 236802.

[2] S. Chen et al., Appl. Phys.Lett. 87 (2005) 263207.

[3] J. Y. Huang et al. Nature 439 (2006) 281. 

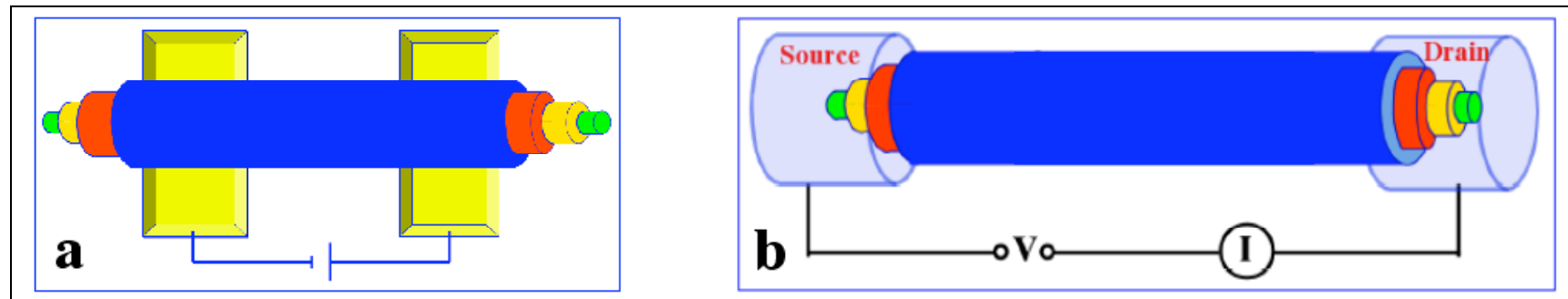

Fig. 1 A schematic drawing showing a side-contact (a) and an end-contact (b) geometry. In the sidecontact configuration, only the outmost wall is contacted to the electrodes, while in the end-contact configuration, each wall is contacted to the electrodes.
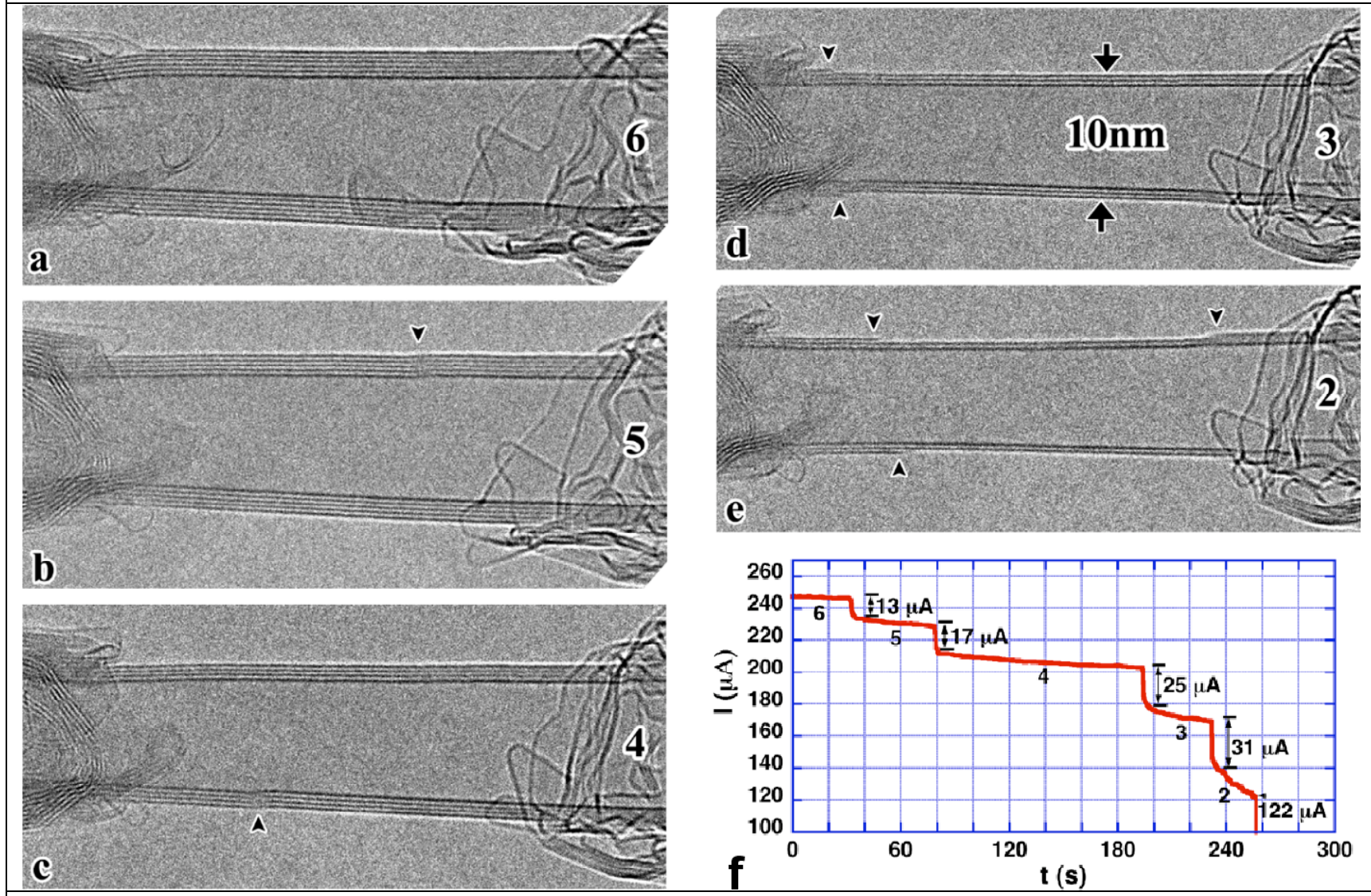

Fig. 2 Sequential HRTEM images showing that the six-wall nanotube is removed wall-by-wall from the outermost wall (a) to the innermost wall (e). The bias voltage is $3 \mathrm{~V}$, and the current flowing in the nanotube is about $240 \mu \mathrm{A}$. The numbers indicate the total number of walls. The arrows mark kinks. The arrowheads denote the residue of the 4 th and the 3 rd walls after breakdown. (f) The elimination of each wall causes a current drop in the I-t curve. 